\title{
On the influence of welding residual stresses on the dynamic behavior of structures
}

\author{
A.C. Bezerra, L.C. Vieira, D.A. Rade* and A. Scotti \\ Federal University of Uberlândia, School of Mechanical Engineering, Campus Santa Mônica, P.O. Box 593, \\ 38400-902, Uberlândia, MG, Brazil
}

Received 2007

Revised 2007

\begin{abstract}
It is widely known that welding processes induce the generation of residual stresses, which, through the so-named stress stiffening effect, can influence the static and dynamic behavior of the welded components. Thus, accounting for this influence becomes important for the understanding of experimental observations and accurate modeling of the dynamic behavior. In this study, the numerical and experimental characterization of the influence of welding residual stresses on the flexural dynamic characteristics of rectangular plates is addressed. It is suggested a general modeling methodology based on finite elements comprising three subsequent analyses, namely: a thermal analysis to compute the transient temperature history due to welding thermal loading; a structural analysis accounting for plastic strains to obtain the welding residual stress fields and geometric distortions, and a dynamic analysis to compute the dynamic characteristics taking into account the stress-stiffening effect and geometric distortions. The results demonstrate the importance of considering the influence of welding residual stresses in the prediction of the flexural dynamic behavior of plates and the feasibility and efficiency of the simplified modeling approach, which can readily be extended to more complex situations, for characterizing this influence.
\end{abstract}

Keywords: Stress stiffening, welding simulation, residual stress, modal analysis

\section{Introduction}

Welding is one of the most widely used manufacturing processes in many branches of industry. It is known that, due to the high temperature gradients involved, this process generates residual stresses. Most frequently, these stresses may jeopardize the quality of welded components since they induce shape distortion and reduction of fatigue strength [1].

A well-known phenomenon, which is called stress-stiffening effect, implies that the static and dynamic behavior of thin structures, such as membranes, columns, plates and shells, can be influenced by the stress state they are subjected to. Many studies have put in evidence this effect, which can be observed in many common systems such as in the tuning mechanisms of musical instruments (string-based and drums).

The coupling between in plane-loads and the flexural dynamic behavior of columns and plates is the basis of the dynamic criterion for elastic stability which has been shown by Bolotin [2] and Lurie [3] to be more general than the static Euler criterion, since, as opposed to the later, it can applied to both conservative and non-conservative structural systems. Such coupling has been explored by Livingston et al. [4] in an inverse procedure intended to identify axial loads in columns, based on Euler-Bernoulli model and the values of natural frequencies associated to bending modes. The axial loads are estimated by using a non-linear least-square technique. More recently, the identification of axial loads and effective clamping stiffness in columns has been carried-out for various boundary conditions using a LifeCycle model optimization algorithm [5].

\footnotetext{
*Corresponding author. E-mail: domingos @ufu.br.
} 
Greening and Lieven studied the influence of residual stresses induced by cold-working of metal plates and imposed pre-stress on the modal properties of a redundant frame structure [6]. Later on, the same authors suggested a procedure for the identification of axial loads acting on the bars of a plane frame by using a finite element model updating procedure in which the axial loads were considered as the correction parameters [7].

Considering also plane frames, Mead [8] analyses in detail the influence of an axial load introduced by heating of one of the members on the free vibrations of the structure. In reference [9], this author investigates the influence of a non-uniform temperature distribution on the buckling and dynamic behavior of thin plates.

In an interesting practical application, in-plane residual stresses were introduced deliberately in band-saws with the purpose of improving cutting performance, as reported by Wang and Mote Jr. [10].

The influence of thermal residual stresses resulting from manufacturing process on the mechanical behavior of composite laminate plates was studied by Almeida and Hansen [11]. The authors suggested the possibility of improving the mechanical characteristics of such structures by a voluntary introduction of thermal residual stresses. Numerical results demonstrated the possibility of achieving increased buckling loads.

Donadon et al. [12] studied the stress-stiffening effect entailed by in-plane stresses produced by piezoelectric patches bonded to the surfaces of laminated composite plates. The sensitivity of the natural frequencies with respect to the applied DC voltage was considered to be significant and the authors suggest some means of improving it.

Simons and Leissa [13] used the Rayleigh-Ritz approach to investigate the influence of in-plane acceleration loads on the natural frequencies of rectangular cantilever plates.

Specifically the case of welding residual stresses, less reports can be found in the literature. Kaldas e Dickinson [14] used the Rayleigh-Ritz approach combined with the finite difference method to model the influence of welding residual stresses in the vibration natural frequencies of rectangular thin plates.

In his Ph.D. thesis Vieira Jr. [15] investigated numerically the influence of welding residual stresses on the natural frequencies of thin metallic plates. In spite of many simplifying assumptions, the modeling strategy based on the Assumed Modes approach has shown to give meaningful predictions as compared to numerical and experimental results provided by other authors. Figure 1(a) illustrates some results presented by this author, which enable to evaluate the influence of welding input power on a typical driving point frequency response function (FRF) of a rectangular plate. For the sake of illustration, the significance of welding residual stress on the dynamic characteristics of rectangular plates is confirmed by the experimental results obtained by the author in Fig. 1(b), in which on can compare the amplitudes of the FRFs of a plate in two conditions: annealed (without residual stresses), and subjected to residual stresses induced by a thermal loading similar to that occurring in TIG welding without added material.

By using the Rosenthal model combined with the Assumed Modes Approach, Vieira Jr. et al. [16] proposed an inverse technique intended to assess information about the level and distribution of welding residual stresses in thin rectangular plates from the dynamic responses. The methodology was validated by numerical applications based on experimentally measured natural frequencies.

More recently, the influence of residual stresses on the dynamic behavior of welded structures was extensively studied by Bezerra [17], who considered various types of structural components (flat thin rectangular plates, reinforced rectangular plates, thin and moderately thick cylinders).

The present paper is intended to report some of the results of the study carried out by Bezerra [17]. The objectives are: i) to suggest and validate a finite element modeling procedure for estimating welding residual stresses and distortions which, as compared to other simplified modeling techniques, can be applied to more complex situations with improved accuracy; ii) to quantify the influence of welding residual stresses on the dynamic behavior of rectangular plates and, as a result, iii) to corroborate previous conclusions of other authors about the necessity of accounting for this influence for accurate modeling.

The welding process considered herein is the TIG process without filler metal and ANSYS ${ }^{\circledR}$ code is used for 3-D modeling.

\section{A review of thermomechanical modeling of welded structures}

To evaluate numerically the influence of residual stresses and distortions on the dynamic behavior accounting for the stress stiffening effect, three analyses must be carried out in succession, namely: $1^{\text {st }}$ ) a transient thermal 


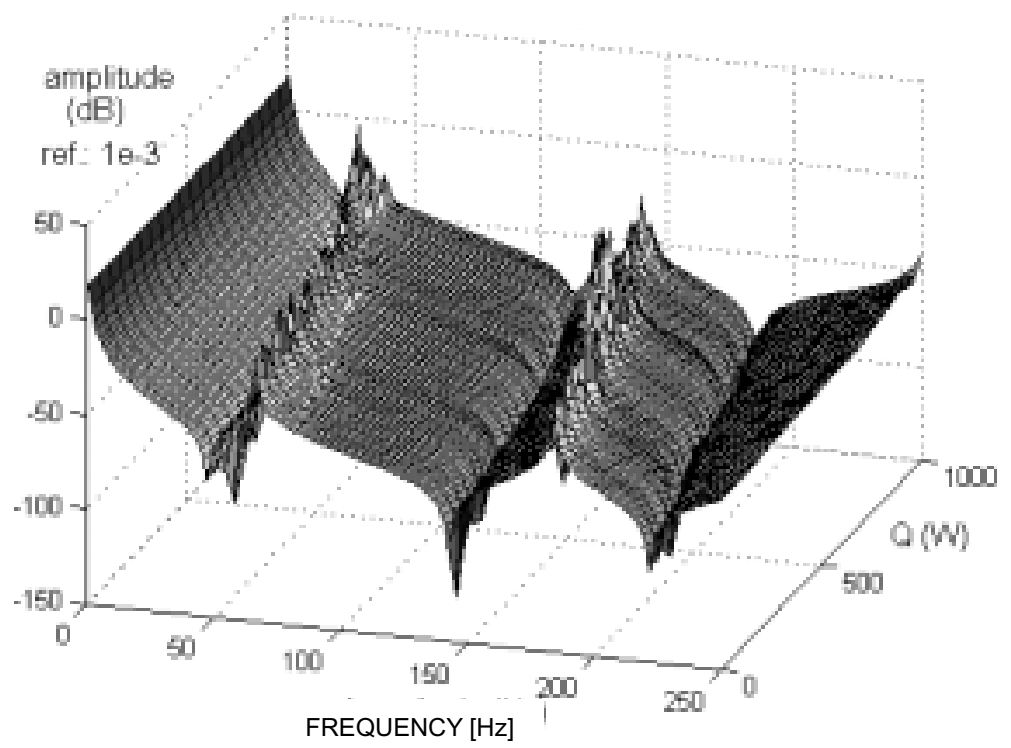

(a)

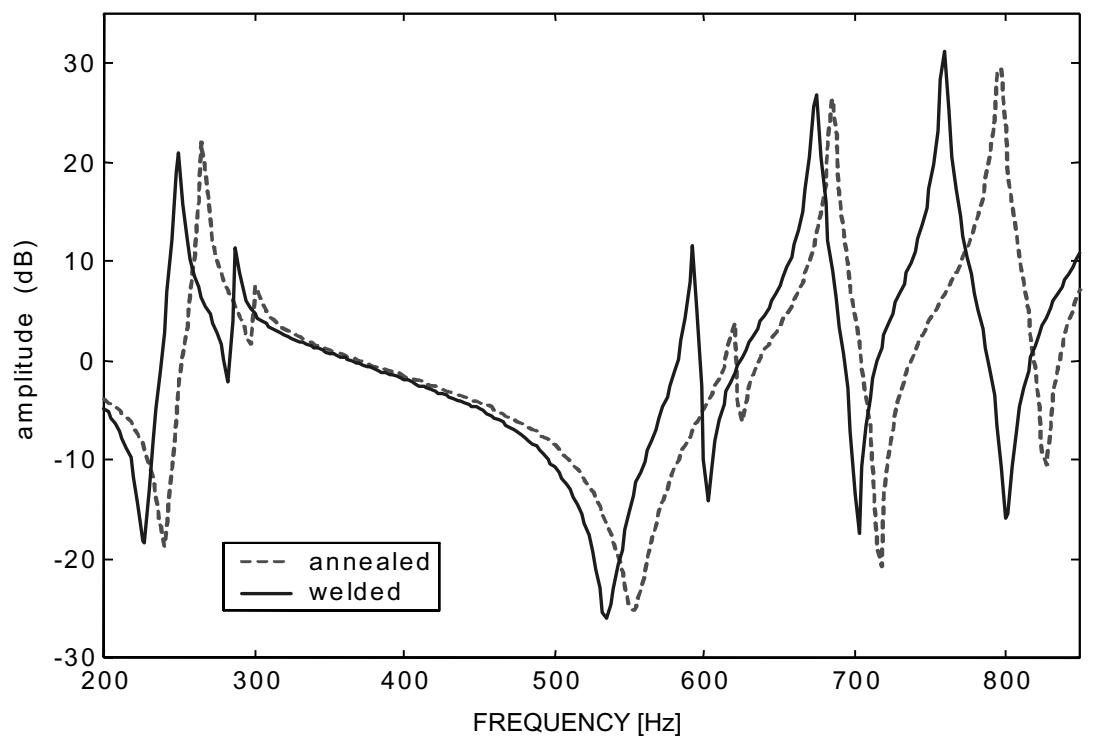

(b)

Fig. 1. (a) amplitudes of a typical numerically simulated driving point FRF for different values of the welding power; (b) amplitudes of an experimentally acquired FRF of a plate in two conditions: without residual stresses (annealed) and with residual stresses (welded) (from Vieira Jr. [15]).

analysis to determine the evolution of temperature over the welded part, given the thermal loading involved in the welding process; $2^{\text {nd }}$ ) a structural analysis intended to obtain the distributions of residual stresses and shape distortions associated to plastic strains induced by the thermal gradients; $3^{r d}$ ) a dynamic analysis (either eigenvalue or frequency response analysis) accounting for the residual stresses, which intervene in the stiffness of the system, and shape variations. 
In the study reported herein, the analyses described above were performed by finite element modeling, using the commercial code ANSYS ${ }^{\circledR}$. The theoretical foundations related to these three analyses are briefly presented in what follows.

\subsection{Thermal analysis}

During TIG welding, the actual heat input per unit of time can be expressed as:

$$
\dot{Q}=\eta U I
$$

where $U$ is the voltage, $I$ is the current and $\eta$ is the arc efficiency.

Heat flow in welding is a non-linear phenomenon owing to the dependence of the material's thermo-physical properties on temperature. The general mathematical model is represented by the three-dimensional heat diffusion equation [18]:

$$
\rho(T) c(T) \frac{\partial T}{\partial t}=q+\frac{\partial}{\partial x}\left(K_{x}(T) \frac{\partial T}{\partial x}\right)+\frac{\partial}{\partial y}\left(K_{y}(T) \frac{\partial T}{\partial y}\right)+\frac{\partial}{\partial z}\left(K_{z}(T) \frac{\partial T}{\partial z}\right)
$$

where $\rho(T)$ is the mass density, $c(T)$ is the specific heat, $q$ is the heat generated per unit volume, $K_{x}(T), K_{y}(T)$ e $K_{z}(T)$ are the thermal conductivity coefficients in each direction, $T$ is temperature and $t$ is time.

Heat losses on the workpiece surfaces by convection, $q_{c}$, and radiation, $q_{r}$, are introduced as boundary conditions using the following equations [18]:

$$
\begin{aligned}
& q_{c}=h\left(T-T_{\infty}\right) \\
& q_{r}=\delta S\left(T^{4}-T_{\infty}^{4}\right)
\end{aligned}
$$

where $h$ and $T_{\infty}$ are the convention coefficient and the temperature of the surrounding fluid, respectively, $\delta$ is the Stefan-Boltzmann constant and $S$ is the radiation area.

At the end of the thermal analysis, the time history of temperature over the volume of the welded workpiece is known.

\subsection{Structural analysis}

The structural problem consists in a static equilibrium analysis for each instant of time considered, in which the total strain is expressed as:

$$
\varepsilon_{\text {Total }}=\varepsilon_{e}+\varepsilon_{\text {therm }}+\varepsilon_{p}
$$

where $\varepsilon_{e}$ is the elastic strain, $\varepsilon_{\text {therm }}$ is the thermal strain and $\varepsilon_{p}$ is the plastic strain.

In the modeling of plastic behavior, it is necessary to define the elastic domain, which is usually based on the von Mises criterion as follows:

$$
f=\sigma_{v M}-\sigma_{y}<0
$$

where $f$ is the yielding function, $\sigma_{y}$ is the material's yield stress and $\sigma_{v M}$ is the von Mises equivalent stress, which is defined as [19]:

$$
\sigma_{v M}=\sqrt{\frac{1}{2}\left[\left(\sigma_{1}-\sigma_{2}\right)^{2}+\left(\sigma_{2}-\sigma_{3}\right)^{2}+\left(\sigma_{3}-\sigma_{1}\right)^{2}\right]}
$$

where $\sigma_{1}, \sigma_{2}$ and $\sigma_{3}$ are the principal stresses. According to the von Mises criterion, plastic strains will be developed when the following condition is satisfied:

$$
f=\sigma_{v M}-\sigma_{y}=0
$$

The hardening rule describes the changing of the yield surface with progressive yielding, so that the conditions (i.e. stress states) for subsequent yielding can be established. Two hardening rules are commonly used: isotropic hardening and kinematic hardening [19]. In isotropic hardening, the yield surface remains centered about its initial 
centerline and its size increases as the plastic strains evolve. Kinematic hardening, on the other hand, assumes that the yield surface remains constant in size, but translates in the stress space with progressive yielding.

When plastic deformation occurs, a flow rule defines plastic increment according to [19]:

$$
d \varepsilon_{p}=\lambda \frac{\partial f}{\partial \sigma}
$$

where $\lambda$ is the plastic multiplier, which determines the amount of plastic straining. The consistency equation, $d f=0$ allows the determination of $\lambda$.

The influence of residual stresses on the mechanical behavior of a structure can be understood by reminding that the total potential energy derives from the strain energy and the work done by the internal stresses, according to the following expression, for the particular case of plates in bending, assumed to be positioned on the plane $x-y$ and subjected to a plane stress state $\left(\sigma_{x}, \sigma_{y}, \tau_{x y}\right)$ [20]:

$$
\begin{aligned}
U\left(\sigma_{x}, \sigma_{y}, \tau_{x y}\right)= & \frac{E h^{3}}{24\left(1-\nu^{2}\right)} \int_{x} \int_{y}\left\{\left[\frac{\partial^{2} w}{\partial x^{2}}+\frac{\partial^{2} w}{\partial y^{2}}\right]^{2}-2(1-\nu)\left[\frac{\partial^{2} w}{\partial x^{2}} \frac{\partial^{2} w}{\partial y^{2}}-\left(\frac{\partial^{2} w}{\partial x \partial y}\right)^{2}\right]\right\} d x d y \\
& +\frac{h}{2} \int_{x} \int_{y}\left[\sigma_{x}\left(\frac{\partial w}{\partial x}\right)^{2}+2 \tau_{x y}\left(\frac{\partial w}{\partial x} \frac{\partial w}{\partial y}\right)+\sigma_{y}\left(\frac{\partial w}{\partial x}\right)^{2}\right] d x d y
\end{aligned}
$$

where $h$ is the thickness of the plate, $\nu$ and $E$ are the material's Poisson ratio and Young modulus, respectively.

Upon finite element discretization based on Eq. (10), the following equations of motion governing the free undamped vibrations of the plate accounting for the residual stresses is obtained:

$$
[M]\{\ddot{q}(t)\}+\left(\left[K_{e}\right]+\left[K_{g}\left(\sigma_{x}, \sigma_{y}, \tau_{x y}\right)\right]\right)\{q(t)\}=\{0\}
$$

where $[M]$ is the mass matrix, $\left[K_{e}\right]$ is the elastic stiffness matrix, $\left[K_{g}\right]$ is the geometric stiffness matrix and $\{q(t)\}$ denotes the vector of nodal displacements.

It is to be noted that the stiffness matrix depends on the stress state of the structure.

\subsection{Eigenvalue analysis}

In this work, the dynamic behavior of the welded structures is characterized by their natural frequencies, which can be obtained by the resolution of the following eigenvalue problem, in which damping is neglected.

$$
\left(\left[K_{e}\right]+\left[K_{g}\right]-\omega_{i}^{2}[M]\right)\{\phi\}_{i}=\{0\}
$$

The equation above clearly puts in evidence the influence of the welding residual stresses on the modal properties of the welded structure.

\section{Finite element modeling}

The modeling procedure whose fundamentals are described in the previous section was implemented using finite elements to simulate the TIG (Tungsten Inert Gas) welding, without filler metal, of a plate of dimensions $370 \times$ $264 \times 6.35 \mathrm{~mm}$ made of aluminum 5052-O. The material properties as functions of temperature used in finite element modeling were provided by Zhu and Chao [21], as shown in Fig. 2. In the absence of more complete information, elastic-perfectly plastic behavior was assumed. The following values were taken for the other relevant physical parameters: convection coefficient: $10 \mathrm{~W} / \mathrm{m}^{2} \mathrm{~K}$, emissivity of 0.75 , Poisson ratio: 0.33 , room temperature: $28^{\circ} \mathrm{C}$, melting temperature: $600^{\circ} \mathrm{C}$.

For the thermal analysis the following elements were used: SOLID70, which is a 3D solid element with 3D thermal conduction capability, and SURF152, which enables to model the convection and radiation effects. For both structural and modal analyses, it was used the element SOLID45, which is a 3D solid element with eight nodes having three degrees of freedom per node (displacements in $x, y$ and $z$ directions). The finite element mesh used for all the three analyses, counting 15.375 nodes and 11.840 elements, is depicted in Fig. 3. 

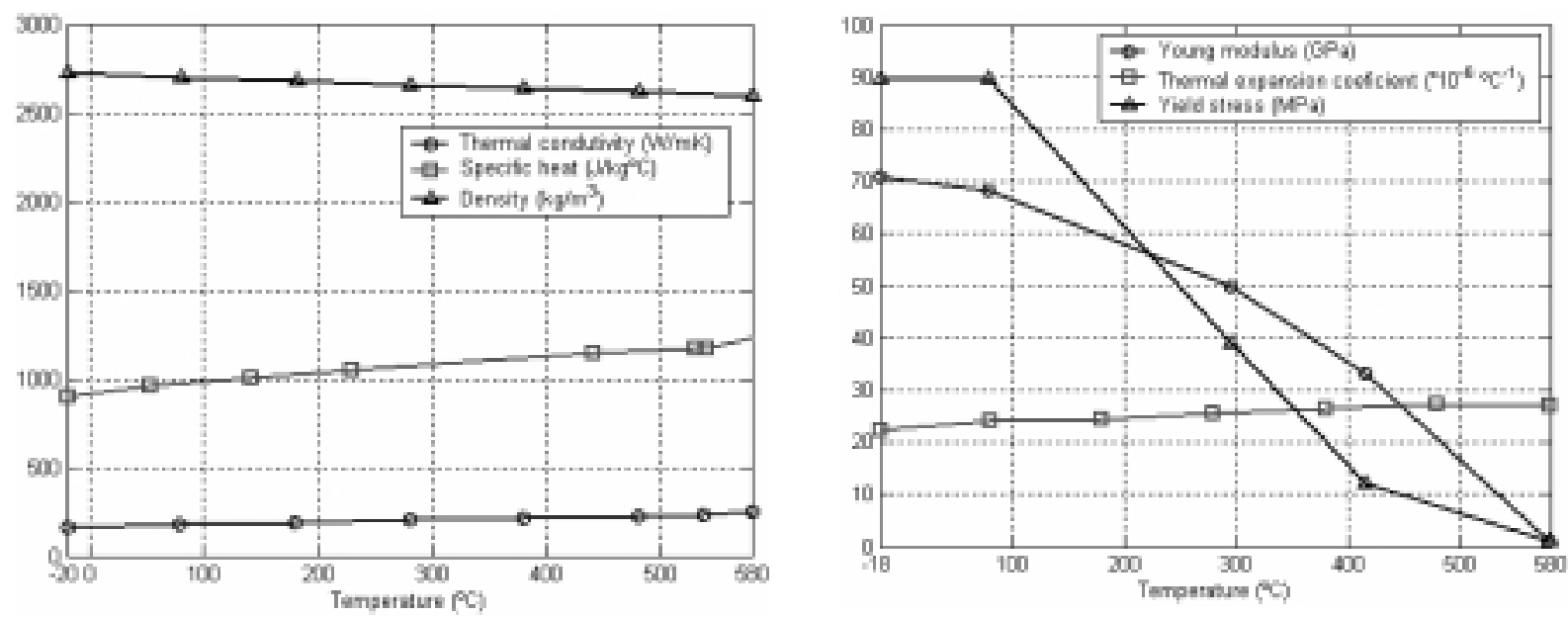

Fig. 2. Physical properties of aluminum 5052 as functions of temperature (adapted from [21]).

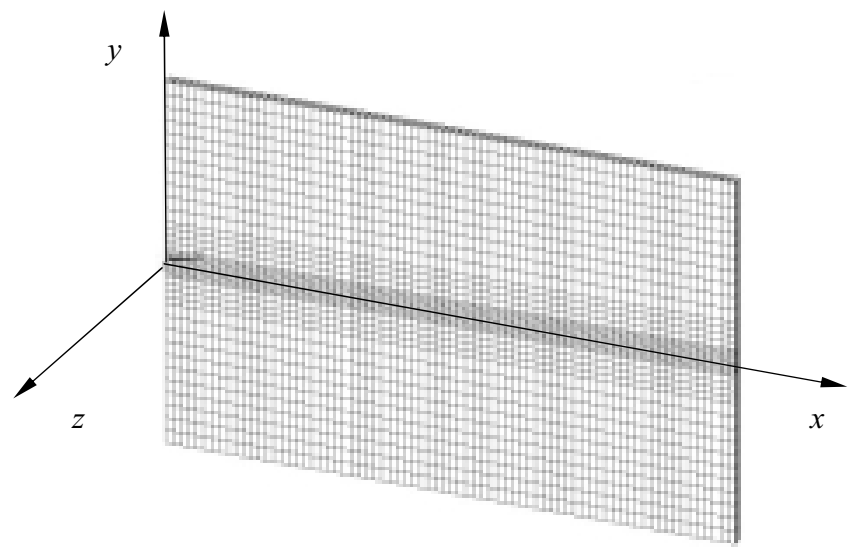

Fig. 3. Finite element mesh of the welded plate.

The relevant welding parameters are those specified in Section 4 for the experimental study, where the values of current and voltage used are the effective values. It was adopted a thermal efficiency of $60 \%$. To account for the volumetric distribution of welding heat, an appropriate model, suggested by Depradeux [22], is used, according to which the heat is introduced in the nodes lying on two planes of finite element model of the plate: the upper face and a parallel plane located $1.59 \mathrm{~mm}$ below it, as depicted in Fig. 4.

\section{Experimental procedure}

The aluminum plate was first heat-treated by annealing at $343^{\circ} \mathrm{C}$ aiming at relieving the residual stresses possibly generated by the manufacturing process. In this heat treatment, care was exercised to avoid geometrical distortions. The plate was welded using TIG process in alternated current (AC) in a single welding pass. The bead was oriented along the longitudinal direction at the centerline of the plate, starting and finishing $20 \mathrm{~mm}$ from each edge. The following welding parameters were used: effective current and voltage: $218 \mathrm{~A}, 12.9 \mathrm{~V}$; argon gas outlet: $10 \mathrm{~L} / \mathrm{min}$; electrode-workpiece distance: $2 \mathrm{~mm}$, welding speed: $20 \mathrm{~cm} / \mathrm{min}$. The torch remained still at the beginning of the weld bead with the arc opened during 7 seconds.

The plate's dynamics was characterized by impact vibration tests before welding and after welding. A set of frequency response functions related to transverse vibrations were obtained by processing the Fourier-transforms of 
Table 1

Values of the natural frequencies of the plate in stress-free and welded condition $(\mathrm{Hz})$

\begin{tabular}{lrrcrc}
\hline Mode $\rightarrow$ & 1 & 2 & 3 & 4 & 5 \\
\hline Stress-free & 210.625 & 241.094 & 493.750 & 495.625 & 611.875 \\
Welded & 170.469 & 204.531 & 445.000 & 507.500 & 548.125 \\
Variation $(\%)$ & -19.065 & -15.165 & -9.87 & 2.40 & -10.42 \\
\hline
\end{tabular}

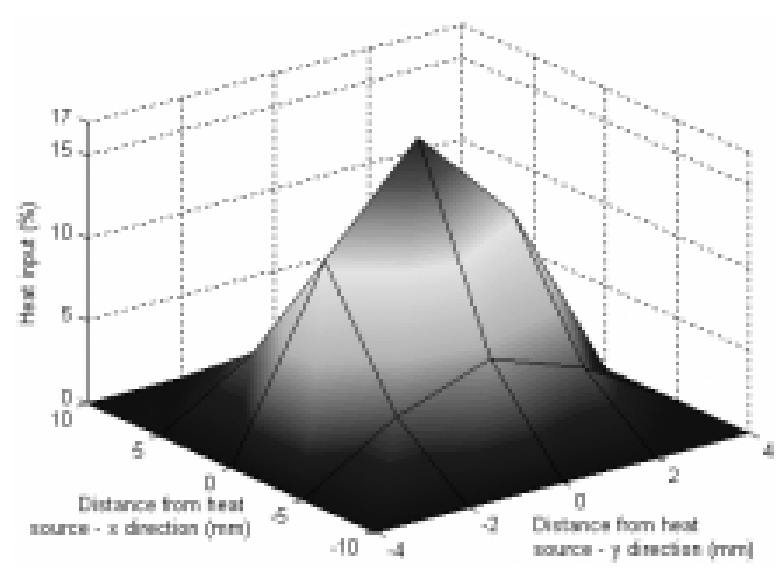

(a)

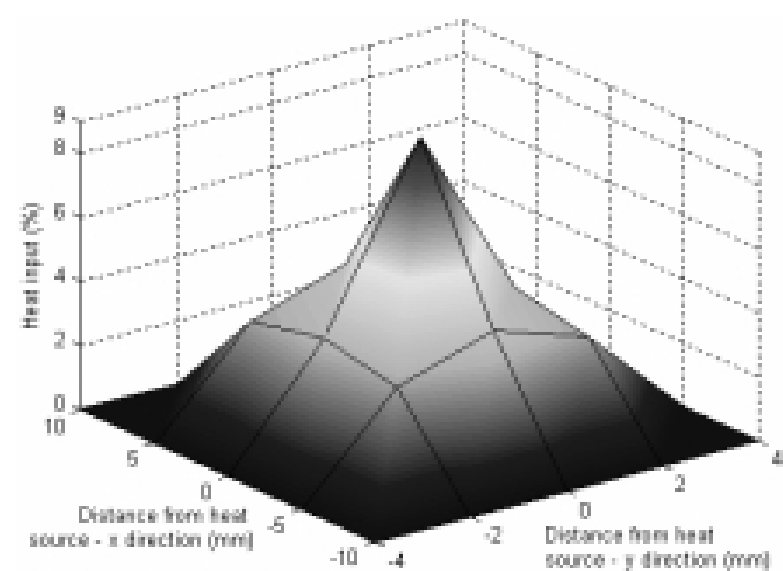

(b)

Fig. 4. (a) heat input distribution on the upper face of the plate; (b) heat input distribution on a plane located $1.59 \mathrm{~mm}$ below the upper surface.
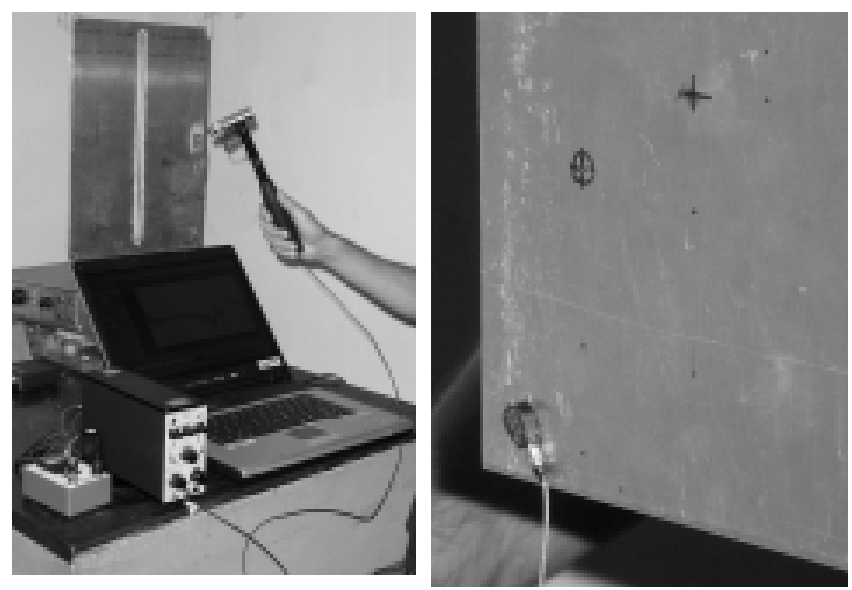

Fig. 5. Experimental setup for the dynamic tests.

input (impact forces) and output (accelerations). The plates were tested suspended by flexible strings, as depicted in Fig. 5.

Figure 6 illustrates the amplitudes of a driving point FRF of the plate in the annealed (stress-free) and welded (with residual stresses) conditions. Table 1 provides the values of the first five natural frequencies for both conditions, as well as the relative variations caused by welding. In spite of the uncertainty in matching the values of the two sets of natural frequencies, it can be seen that the general trend observed is the decrease of the values of the natural frequencies as the result of welding, except for the forth natural frequency. Moreover, it is clearly perceived that the sensitivity of the natural frequencies with respect to the welding residual stresses combined with shape distortions varies from one mode to the other. The observed increase or decrease of the values of the natural frequencies, as well 

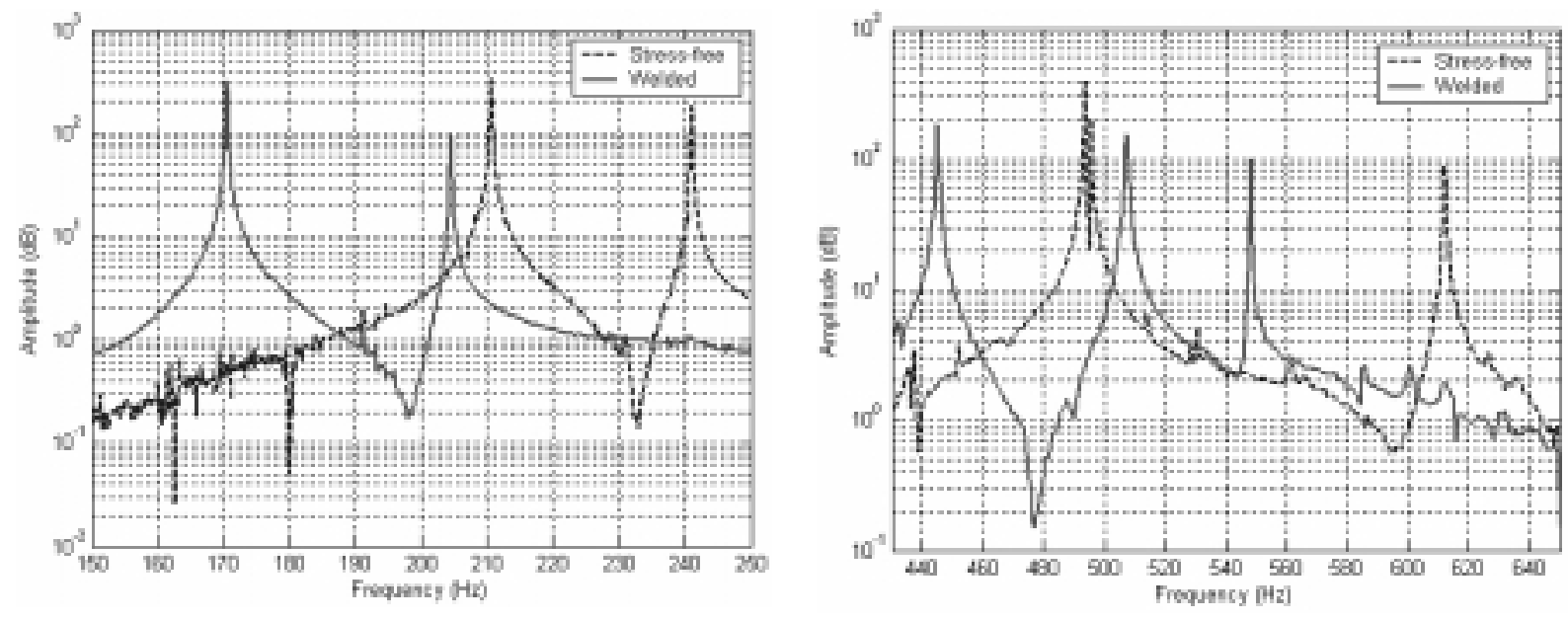

Fig. 6. Amplitudes of a driving-point FRFs of the stress-free and welded conditions.

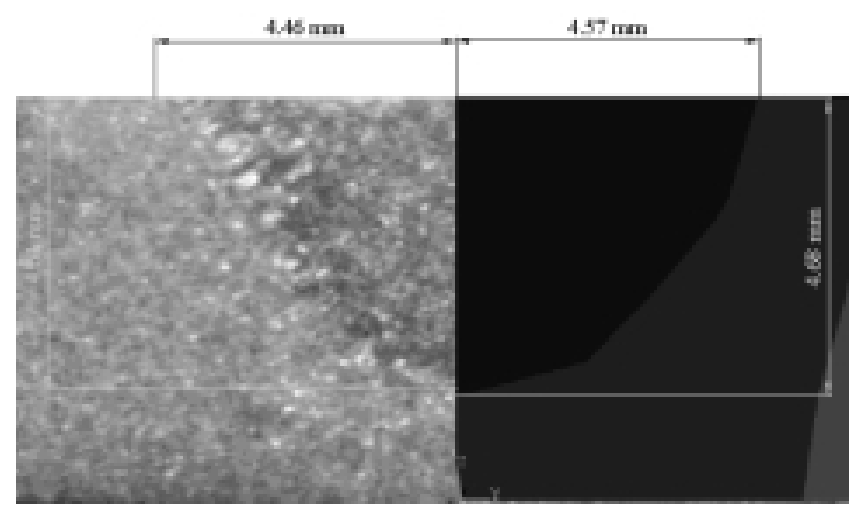

Fig. 7. Comparison of numerical and experimental fusion zones at cross-section $x=170 \mathrm{~mm}$.

as the magnitude of their variations, can be understood by reasoning in terms of modal strain energies, considering the predominance of tensile or compressive normal stresses and the strain distribuitions obtained for each mode shape. In reference [16], in a which a case similar to the one focused herein is addressed, the distributions of normal and shear stresses along longitudinal and transverse sections of the welded plate are presented. Such distributions, together with the deformed shapes (such as those presented in Fig. 10), are helpful in understanding the trends of the variations observed for the natural frequencies. Clearly, the actual variation of each natural frequency is determined by the integral of the strain energy associated to both normal and shear stresses and strains, for each corresponding mode shape.

\section{Comparision between numerical and experimental results}

The results obtained in the thermal analysis are compared to a macrograph obtained experimentally in Fig. 7 , which shows the numerically-predicted and experimentally observed fusion zones at the cross-section located at $x=170 \mathrm{~mm}$ (axes are defined in Fig. 3). It can be verified that the fusion zone has been fairly accurately predicted by the numerical modeling, which errors of $2.5 \%$ for the width and $1.5 \%$ for the depth of the welding pool.

The residual stress field on longitudinal direction is illustrated in Fig. 8 as a contour plot. As expected, it is confirmed the occurrence of high tensile stresses in the longitudinal direction near the weld bead. 
Table 2

Variations of natural frequencies due to welding obtained experimentally and numerically

\begin{tabular}{cccc}
\hline Mode & Experimental $(\%)$ & Numerical $(\%)$ & Deviation \\
\hline 1 & -19.07 & -22.35 & -3.29 \\
2 & -15.17 & -17.75 & -2.58 \\
3 & -9.87 & -11.24 & -1.37 \\
4 & 2.40 & 2.66 & 0.26 \\
5 & -10.42 & -12.17 & -1.75 \\
6 & 4.31 & 4.88 & 0.57 \\
7 & -5.70 & -6.54 & -0.84 \\
8 & -1.83 & -2.01 & -0.18 \\
\hline
\end{tabular}
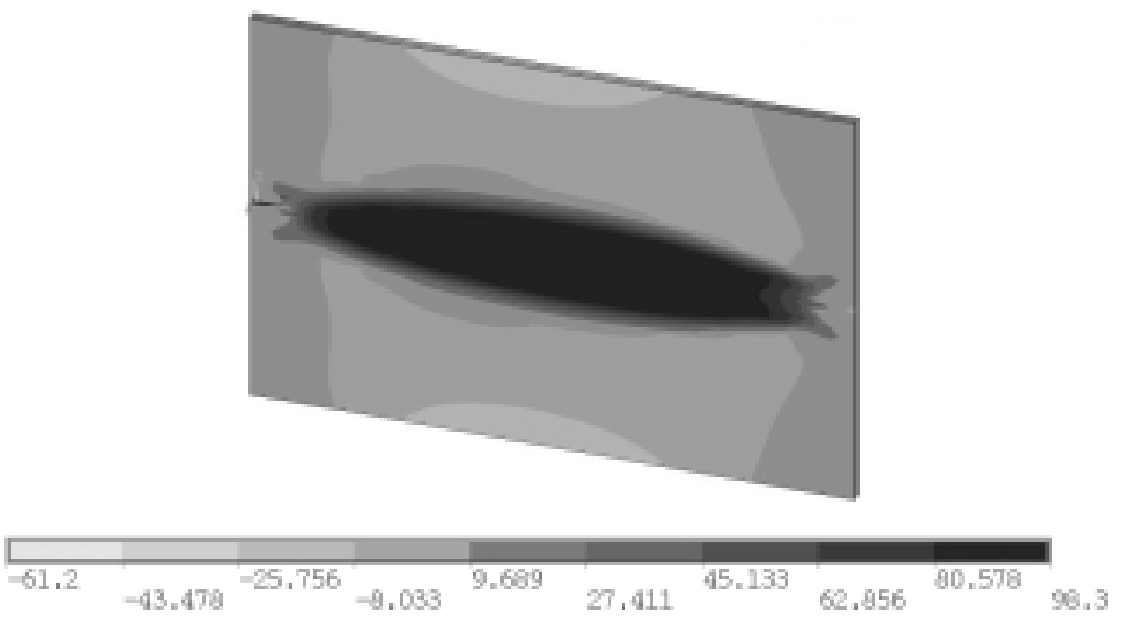

Fig. 8. Residual stress field on longitudinal direction (values in MPa).

Figure 9 illustrates a comparison between experimental and numerical values for residual transverse displacements, that is to say, those that remain after complete cooling of the welded piece, for section $x=50 \mathrm{~mm}$ (Fig .8(a)) and section $y=112 \mathrm{~mm}$ (Fig. 8(b)), both on the upper face of the plate. The experimental transverse displacements were measured using inductive proximity sensors. It can be observed a satisfactory agreement between numerical and experimental results, except for $y=0$ on section $x=50 \mathrm{~mm}$ (Fig. 9(a)), where the numerical result overestimates the displacement. The numerical and experimental results show that shape distortions are very small as compared to the plate dimensions.

Table 2 presents the variations of natural frequencies due to welding, which include both residual stress and distortion effects. It can be seen that the numerical results show the same trend observed in experimental data and that deviations between the values of both sets of natural frequencies are small. Figure 10 shows the first six vibrating mode shapes of the plates without residual stresses. By examining the deformed shapes, and taking into account the distributions of the residual stresses, the different sensitivities of the natural frequencies with respect to those stresses can be qualitatively interpreted. This point will be subject of future studies.

At this point it is interesting to evaluate the influence of residual stresses and distortions on the first vibration mode shapes which are depicted in Fig. 10. For this purpose, the values of the MAC (Modal Assurance Criterion) were computed for each pair of modes obtained from the finite element model, according to the expression:

$$
M A C_{i j}=\frac{\left.\left({ }_{F} \psi_{i}\right\}^{T}\left\{{ }_{S} \psi_{j}\right\}\right)^{2}}{\left.\left.\left.\left\{{ }_{{ }_{F}} \psi_{i}\right\}^{T}\left\{{ }_{F} \psi_{i}\right\}\right\}\left({ }_{S} \psi_{j}\right\}^{T}{ }_{S_{S}} \psi_{j}\right\}\right)} \quad i, j=1 \text { to } 6
$$

where $\left\{{ }_{F} \psi_{i}\right\}$ denotes the $\mathrm{i}$ - $t h$ mode shape of plate in the stress-free state and $\left\{{ }_{S} \psi_{j}\right\}$ indicates the jth mode shape of the plate with residual stresses. It must be noted that the MAC values were computed using all the coordinates of 


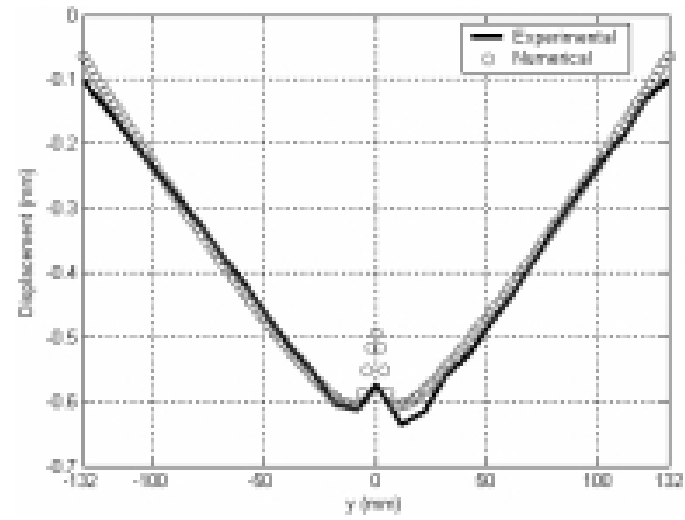

(a)

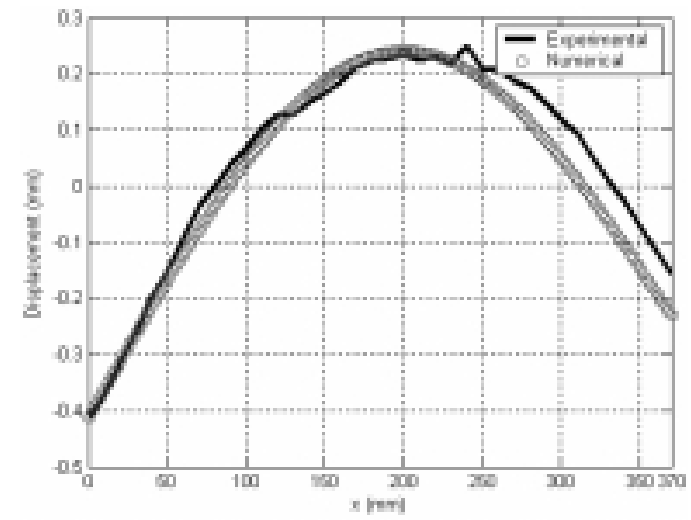

(b)

Fig. 9. Final deformed shape on the upper face of plate 1 of sections $x=50 \mathrm{~mm}$ (a) and $y=112 \mathrm{~mm}$ (b).

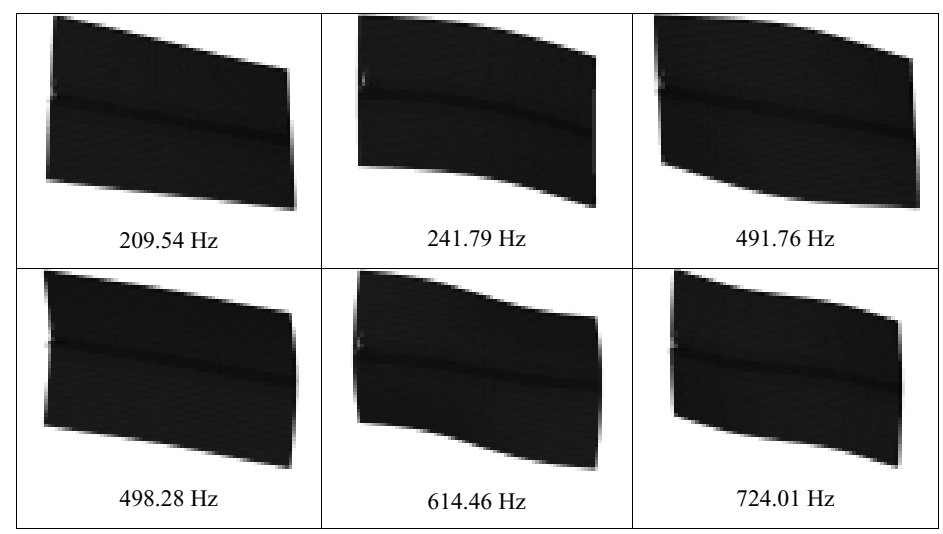

Fig. 10. The first six vibrating mode shapes of the plate without residual stresses.

the eigenvectors, which correspond to the total number of degrees-of-freedom of the structural finite element model (43,125 d.of.).

The matrix formed from the MAC values is shown below, revealing that the mode shapes change very little when residual stresses and distortions are considered.

$$
M A C=\left[\begin{array}{llllll}
0.9999 & 0.0000 & 0.0000 & 0.0000 & 0.0000 & 0.0000 \\
0.0000 & 0.9682 & 0.0000 & 0.0452 & 0.0000 & 0.0000 \\
0.0000 & 0.0000 & 0.9989 & 0.0000 & 0.0000 & 0.0000 \\
0.0000 & 0.0452 & 0.0000 & 0.9760 & 0.0001 & 0.0000 \\
0.0000 & 0.0000 & 0.0000 & 0.0001 & 0.9965 & 0.0006 \\
0.0000 & 0.0000 & 0.0000 & 0.0000 & 0.0006 & 0.9881
\end{array}\right]
$$

To evaluate separately the influence of welding distortions on the values of the natural frequencies of the plate, and to confirm that the observed variations of the natural frequencies can be primarily ascribed to residual stresses, another modal analysis was carried out discarding the stress stiffening effect in the finite element model (which means disregarding the geometric stiffness matrix in Eq. (11)). Table 3 presents the variations of the values of the natural frequencies caused only by geometric distortions. It can be noticed that for almost all modes, the influence of distortion is small as compared to the influence of stress-stiffening induced by the residual stresses. 
Table 3

Variations of the natural frequencies due to welding distortion

\begin{tabular}{lllllllll}
\hline Mode & 1 & 2 & 3 & 4 & 5 & 6 & 7 & 8 \\
\hline Variation $(\%)$ & 0.71 & 1.16 & 0.59 & 1.24 & 0.66 & 0.63 & 0.43 & 0.50 \\
\hline
\end{tabular}

\section{Concluding remarks}

This paper presented experimental and finite element predictions of the influence of geometrical distortions and stress stiffening associated to welding residual stresses on the dynamic characteristics of welded plates. It was suggested a modeling procedure consisting of three successive thermal, structural and dynamic analyses which is capable of properly simulating such influence and are well adapted to be applied to more complex situations, such as those involving structures of other geometries.

In the case considered it was observed that the contribution of welding distortions on the changes in natural frequencies is small as compared to the effect of stress stiffening, which will not be always the case. The modeling methodology enables to evaluate separately each of the influences, though in practice both will exist simultaneously.

In spite of the simplifying assumptions adopted in the modeling, the results of numerical simulations have shown to be in good agreement with experimental ones. It should be noted that accounting for metallurgical transformations of the welded material would bring great complexity to the modeling. However, in the case studied, such effect could be disregarded since significant metallurgical transformations are not expected for the aluminum alloy considered. In other situations in which phase transformations are expected to be relevant, the capability of some specific commercial finite element software specialized in the modeling of thermo-mechanical treatments can be explored.

From the standpoint of dynamic behavior, both the simulations and experiments have demonstrated that the welding residual stress can have a significant influence on the dynamic characteristics of the welded plates. Numerical simulations performed on other types of structural components have demonstrated that such influence is more important in the case of thin plate-like or shell-like structures. Thus, it becomes important, in certain cases, to account for welding residual stresses in the characterization of the dynamic behavior of such structures, specially in applications such as finite element model updating and assessment of response variability.

\section{Acknowledgements}

The authors wish to thank Brazilian agencies CNPq and CAPES for the grant of doctorate and research scholarships. The support of agencies FAPEMIG through project TEC317/03 and CNPq through project 477600/2004-2 is gratefully acknowledged.

\section{References}

[1] A.J.A. Parlane, J.S. Allen, J.D. Harrison, R.H. Leggatt, Dwight, J.B., Bailey, N., Procter, E. and Saunders, G.G., "Residual Stresses and their Effect", The Welding Institute, U.K., 1981.

[2] V.V. Bolotin, Non Conservative Problems of the Theory of Elastic Stability, Pergamon Press Ltd., 1963.

[3] H. Lurie, Lateral Vibrations as Related to Structural Stability, Journal of Applied Mechanics 19 (1951), $195-204$.

[4] Livingston, J.G. Béliveau, D.R. Huston, Estimation of axial load in prismatic members using flexural vibrations, Journal of Sound and Vibration 179(5) (1995), 899-908.

[5] J.E.R. Flores, F.A.C. Viana, D.A. Rade and V. Steffen Jr., Identification of External Forces in Mechanical Systems by Using LifeCycle Model and Stress-Stiffening Effect, to appear in Mechanical Systems and Signal Processing, 2007.

[6] N.A.J. Lieven and P.D. Greening, Effect of Experimental Pre-Stress and Residual Stress on Modal Behavior, Phil Trans R Soc Lond A 359 (2001), 97-111.

[7] N.A.J. Lieven and P.D. Greening, Identification and Updating of Loading in Frameworks Using Dynamic Measurements, Journal of Sound and Vibration 260(1) (2003), 101-115.

[8] D.J. Mead, Free Vibrations of Self-strained Assemblies of Beams, Journal of Sound and Vibration 249(1) (2002), 101-s115.

[9] D.J. Mead, Vibration and Buckling of Flat Free-Free Plates under Non-uniform In-plane Thermal Stresses, Journal of Sound and Vibration 260(1) (2003), 141-165.

[10] J. Wang and C.D. Mote Jr., The Effect of Roll-Tensioning on Bandsaw Plate Vibration and Stability, Journal of Sound and Vibration 175(5) (1994), 677-692. 
[11] S.F.M. Almeida and J.S. Hansen, Enhanced Elastic Buckling Loads of Composite Plates With Tailored Thermal Residual Stresses, Journal of Applied Mechanics 64 (1997), 772-780.

[12] M.V. Donadon, S.F.M. Almeida and A.R. Faria, Stiffening Effects on the Natural Frequencies of Laminated Plates with Piezoelectric Actuators, Composites Part B-Engineering 33(5) (2002), 335-242.

[13] D.A. Simons and A.W. Leissa, Vibrations of Rectangular Cantilever Plates Subjected to In-Plane Acceleration Loads, Journal of Sound and Vibration 17(3) (1971), 407-422.

[14] M.M. Kaldas and S.M. Dickinson, The Flexural Vibration of Welded Rectangular Plates, Journal of Sound and Vibration 75(2) (1981), $163-178$.

[15] A.B. Vieira Jr, Identification of Stresses in Rectangular Plates from Dynamic Responses, with Application to Welding Residual Stresses, $\mathrm{Ph} . \mathrm{D}$. Thesis, School of Mechanical Engineering, Federal University of Uberândia, 2003 (in Portuguese).

[16] A.B. Vieira Jr., D.A. Rade and A. Scotti, Identification of Welding Residual Stresses in Rectangular Plates Using Vibration Responses, Inverse Problems in Engineering, England 14(3) (2006), 313-331.

[17] A.C. Bezerra, Numerical Simulation of Welding with Application to the Characterization of the Dynamic Behavior of Welded Structures, $\mathrm{Ph} . \mathrm{D}$. Thesis, School of Mechanical Engineering, Federal University of Uberândia, 2006 (in Portuguese).

[18] F.P. Incropera and D.P. de Witt, Fundamentals of Heat and Mass Transfer, 3rd edition, John Wiley, 1990.

[19] J. Lemaitre and J.L. Chaboche, Mécanique des Matériaux Solides, Dunod, 2nd edition, 1988 (in French).

[20] S. Timoshenko, Theory of Elastic Stability, First Edition, McGraw-Hill, New York, 1936.

[21] X.K. Zhu and Y.J. Chao, Effects of Temperature-Dependent Material Properties on Welding Simulation, Computers and Structures 80 (2002), 967-976.

[22] L. Depradeux, Simulation Numérique du Soudage - Acier 316L - Validation sur Cas Tests de Complexite Croissante (Numerical Simulation of Welding - Steel 316L - Validation on Test-cases of Increasing Complexity), Doctorate Thesis, Institut National des Sciences Appliqúes de Lyon, France, 2004 (in French). 

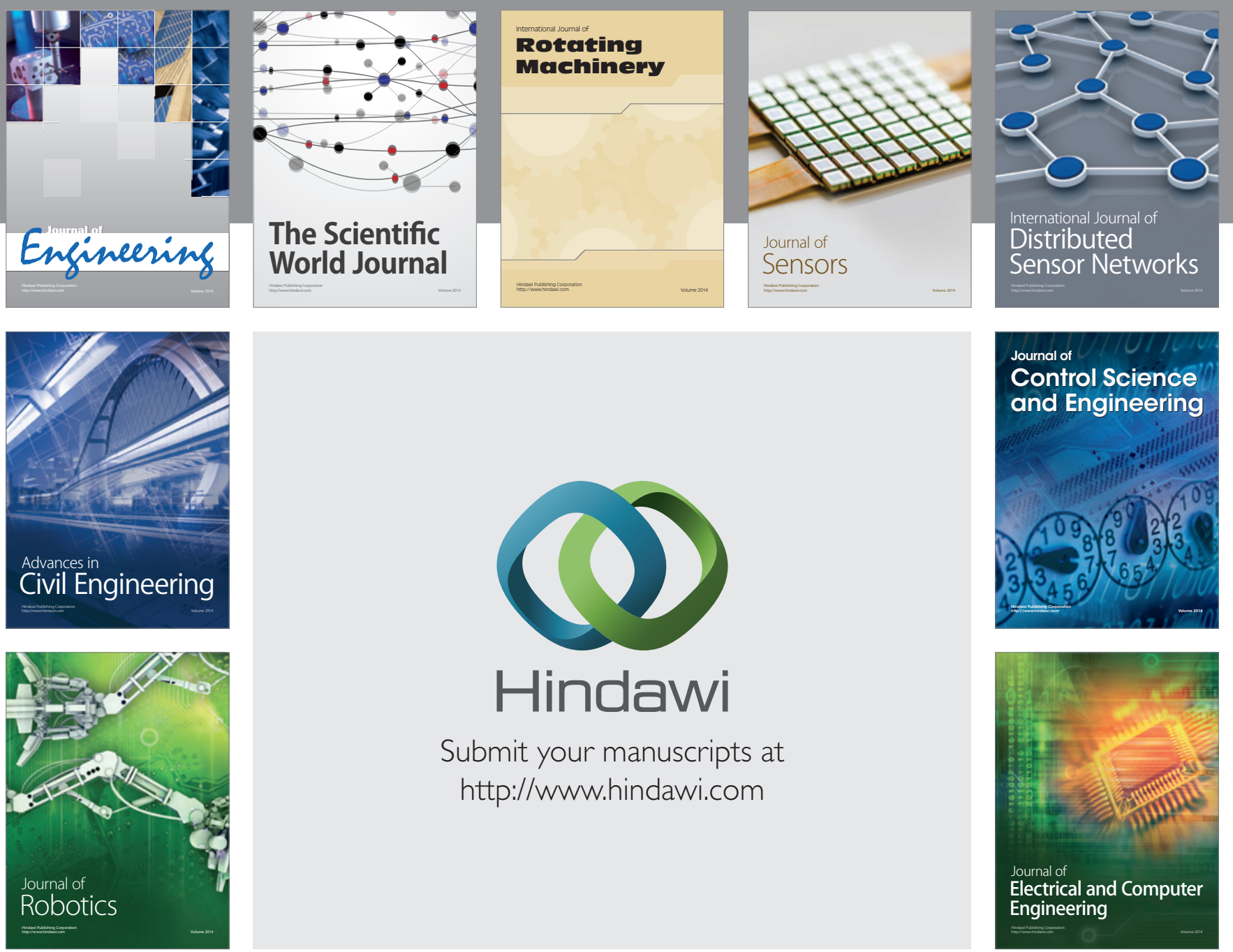

Submit your manuscripts at

http://www.hindawi.com
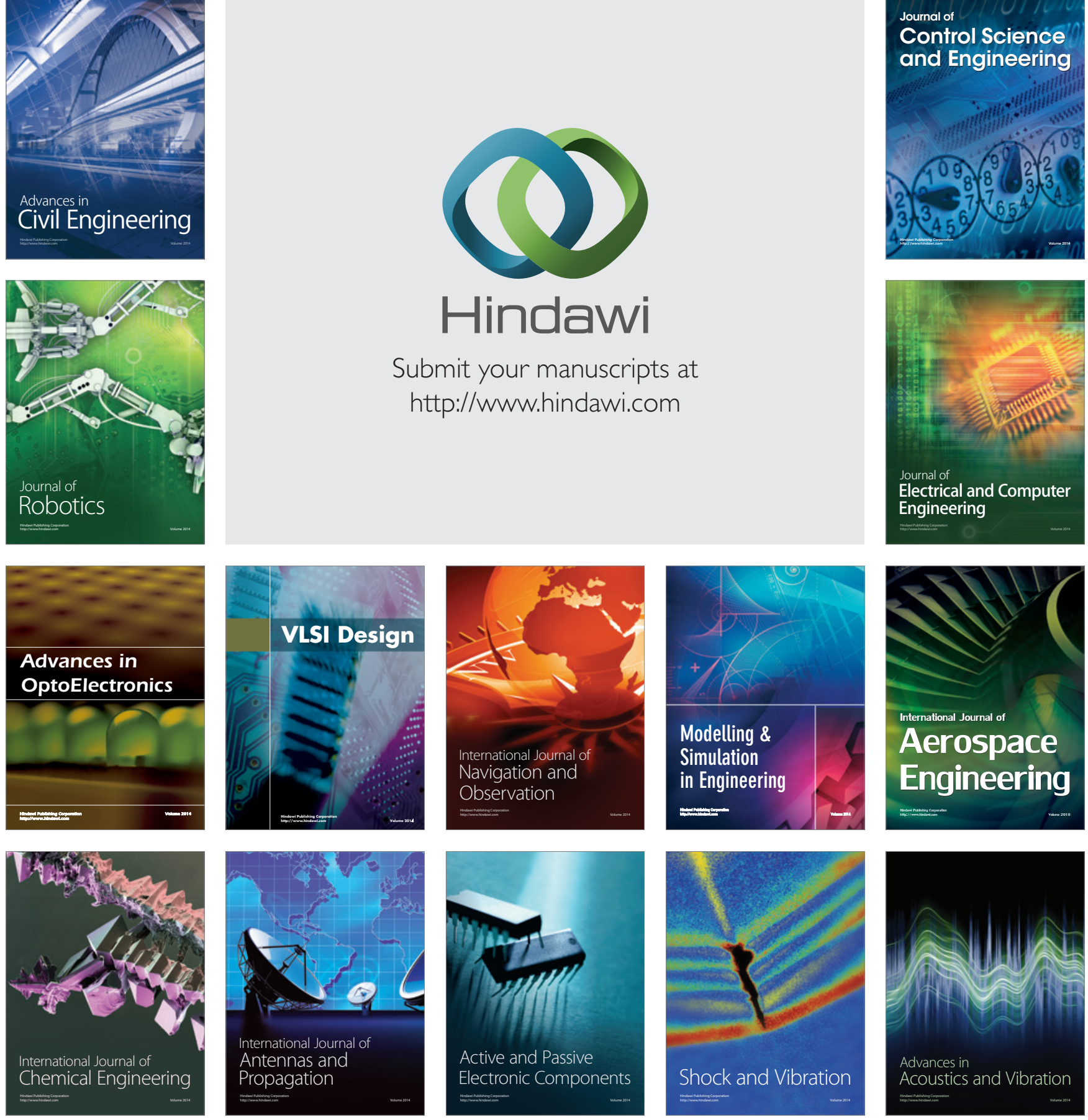